\title{
The Research of the Technology for the Cascaded H Bridge Converter to Eliminate Specific Harmonic of the Step Form
}

\author{
Yu Zhang \\ School of North China Electric Power University, Baoding 071000, China \\ 763446638@qq.com
}

Keywords: SHEPWM, Monte Carlo, nonlinear programming.

\begin{abstract}
This paper mainly studies and analyzes the output voltage wave which is based on the control of step form SHEPWN. We solve the Switching Angles in the condition that the number of $\mathrm{H}$ bridge converter unit is $\mathrm{n}=3$ and that the modulation ratio amplitude is $m=0.8$. We solve the problem by changing the nonlinear equations to nonlinear programming and set up the initial value by the Monte Carlo random sampling. We determine there are outliers in the range of $\mathrm{m}$ by the computer simulation, so we can not simply determine the rang of $\mathrm{m}$ by solving the maximum and minimum of $\mathrm{m}$. At the same time, we get the maximum range of $\mathrm{m}$ by theoretical argument and consulting materials. Therefor we enumerate $\mathrm{m}$ with certain step length and try to solve the equation set. And we get the range of $\mathrm{m}$ and calculate the corresponding THD in this way.
\end{abstract}

\section{The solution of the Switching Angles and the calculation of THD}

When the $\mathrm{n}$ is 3 and the $\mathrm{m}$ is 0.8 , we solve the equation set as follows.

$$
\left\{\begin{array}{c}
\cos \theta_{1}+\cos \theta_{2}+\cos \theta_{3}=\frac{3 \pi}{5} \\
\cos 5 \theta_{1}+\cos 5 \theta_{2}+\cos 5 \theta_{3}=0 \\
\cos 7 \theta_{1}+\cos 7 \theta_{2}+\cos 7 \theta_{3}=0
\end{array}\right.
$$

Among this $0 \leq \theta_{1}<\theta_{2}<\theta_{3} \leq \pi / 2$.

We care that all switch angles which are $\theta_{1}, \theta_{2}$ and $\theta_{3}$ are symmetric. So we can change the inequality constraints to $0 \leq \theta_{i} \leq \pi / 2 i=1,2,3$ and order the angles from small to large. Then we change the equation set to nonlinear programming problem: the programming object is to minimize the Root Mean Square(RMS) of the original equation set error and the restrictions are the original restrictions. The nonlinear programming is as follow:

$$
\min \sqrt[2]{\frac{\left(\vartheta_{1}-\frac{3 \pi}{5}\right)^{2}+\left(\vartheta_{2}-0\right)^{2}+\left(\vartheta_{3}-0\right)^{2}}{3}}
$$

Among this

$$
\theta_{k}=\sum_{i=1}^{n} \cos k \theta_{i}
$$

We draw help from the fmincon function in the Matlab to solve this nonlinear problem with restrictions. Now the problem we is facing is how to establish the initial value $\theta^{\circ}$ : if the selection of initial value is not appropriate will cause the solving process of the equation set is not convergent or converges in the local optimal solution and the result is the failure of the solution. On the other hand the selection of the appropriate initial value can reduce the number of iteration. Now there is not appropriate way to set the initial value through consulting some information. And existing theoretic basis for the initial value setting usually is applicable to the situation that $\mathrm{n}$ is less. The empirical way can not solve satisfied solution ${ }^{[1]}$. We notice that at this time the scale of the problem is small. In once solution we can repeatedly choose random initial value into solution by the Monte Carlo sampling and then choose the best as the final solution. Because the unknown numbers only have 
three and $0 \leq \theta_{i} \leq \pi / 2$, the little number of sampling can satisfy the require and we choose to do 30 Monte Carlo sampling.

Objective function which is error of the equation root mean square is $7.3765 \times 10^{-7}$, and it can be accepted. So the switching angles are

Bring $\theta_{1}, \theta_{2}$ and $\theta_{3}$ into original equation set and calculate ${ }^{[4]}$.

$$
\begin{gathered}
\cos \theta_{1}+\cos \theta_{2}+\cos \theta_{3}=1.8850 \approx \frac{3 \pi}{5} \\
\cos 5 \theta_{1}+\cos 5 \theta_{2}+\cos 5 \theta_{3}=-7.7845 \times 10^{-7} \approx 0 \\
\cos 7 \theta_{1}+\cos 7 \theta_{2}+\cos 7 \theta_{3}=-7.7557 \times 10^{-7} \approx 0
\end{gathered}
$$

Obviously the switching angles agree with original equation set.

According to the solution and corresponding formula we can calculate the THD is $11.86 \%$.

\section{The Range of $\mathbf{m}$}

We use grid method to enumerate $\theta_{1}$ and $\theta_{2}$ and use the Equation 4,5 to calculate $\theta_{3}^{\prime}$ and $\theta_{3}^{\prime \prime}$. If $\theta_{3}^{\prime}=\theta_{3}^{\prime \prime}$ and the imaginary part of $\theta_{3}^{\prime}$ is zero and the real part is greater than zero, we can think there are feasible solutions with $\theta_{1}, \theta_{2}$ and $\theta_{3}$ among $\theta_{3}=\theta_{3}^{\prime}=\theta_{3}^{\prime \prime}$. Then we can calculate corresponding feasible solution $m$ with Equation 6. If the grid density is enough great, we can think all calculating $\mathrm{m}$ together constitute the range of the modulation ratio amplitude which makes the equation set has a solution.

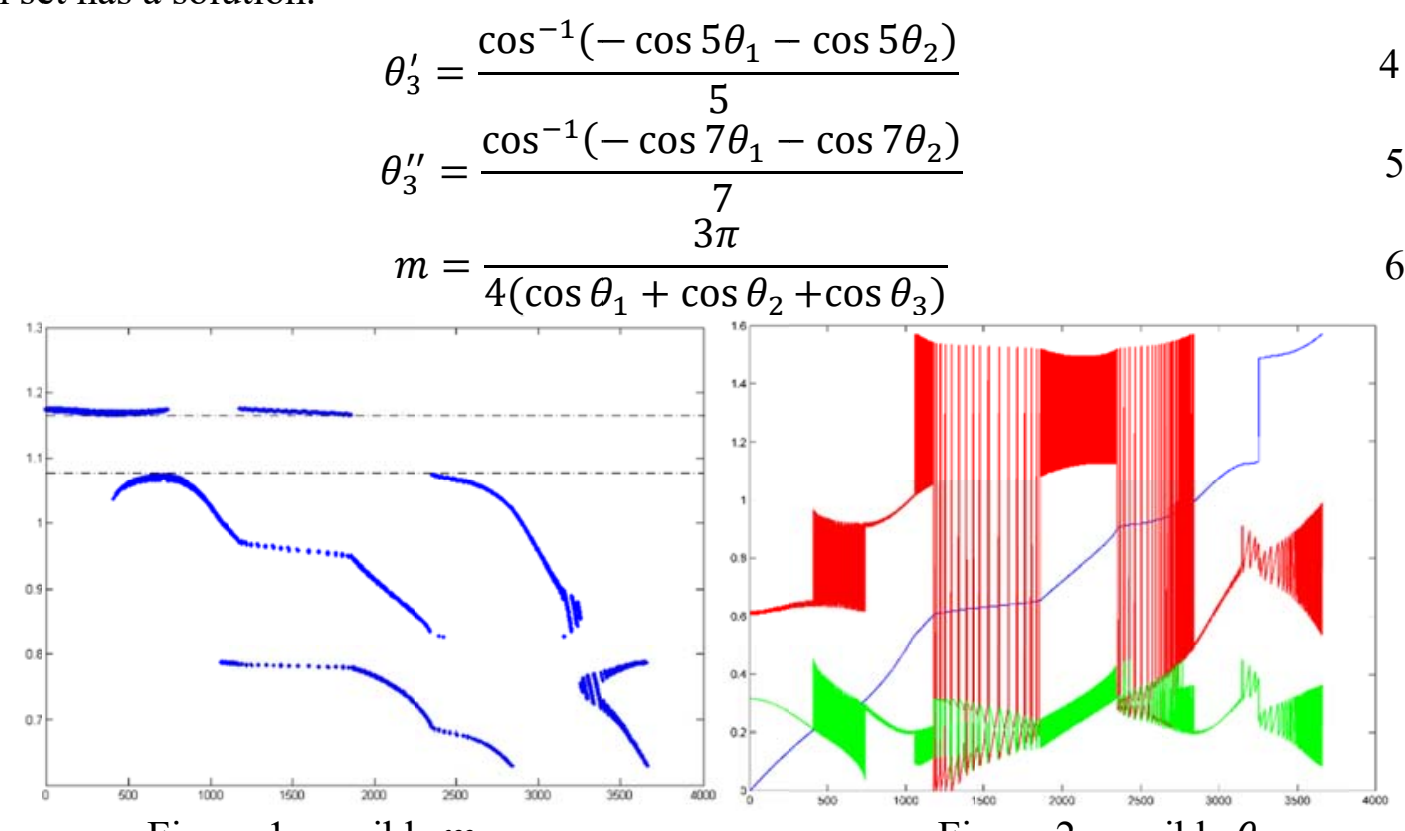

Figure 1 possible $m$

Figure 2 possible $\theta$

The horizontal coordinate is the grid number in above figures. The vertical coordinate is the possible value of $\mathrm{m}$ in Fig.2 and the red, blue and green lines separately represent the possible value of $\theta_{1}, \theta_{2}$ and $\theta_{3}$. The $R_{m}$ is discontinuous by observing that m between 1.07 and 1.16 is not valid value. So we can get $R_{m}$ by solving the maximum and minimum of $\mathrm{m}$.

Therefor we choose the method which enumerates $m$ with certain step length and try use above method to solve corresponding m equation set. If the RMSE of the optimum solution is enough little, we can think there is a valid value in the corresponding $\mathrm{m}$ neighborhood. According to the definition, obviously there is $m>0$, then we get the upper bound of $m$ by theoretical derivation and accessing to information ${ }^{[2]}$.

The theoretical certification is as follow:

$$
V_{k}= \pm \frac{4 V_{d c}}{k \pi} \sum_{i=1}^{n} \cos \left(k \theta_{i}\right), \quad k \text { is the odd number. }
$$


Then by the original equation we know:

$$
V_{1}=\frac{\pi n}{4} m \times \frac{4 V_{d c}}{k \pi}=n V_{d c} m
$$

If $\theta_{1}=\theta_{2}=\cdots=\theta_{n}$, the $V_{1}$ has the maximum at this time which is $\frac{4 n V_{d c}}{\pi} \approx 1.27$.

But generally this situation is meaningless because it can not eliminate any harmonic component under this situation. By accessing to information we usually think the upper bound is 1.15.

We enumerate $\mathrm{m}$ in the region $[0,1.15]$ with the step length $1 \times 10^{-3}$ to try to solve the equation set and draw the relation figure of RMSE and $\mathrm{m}$.

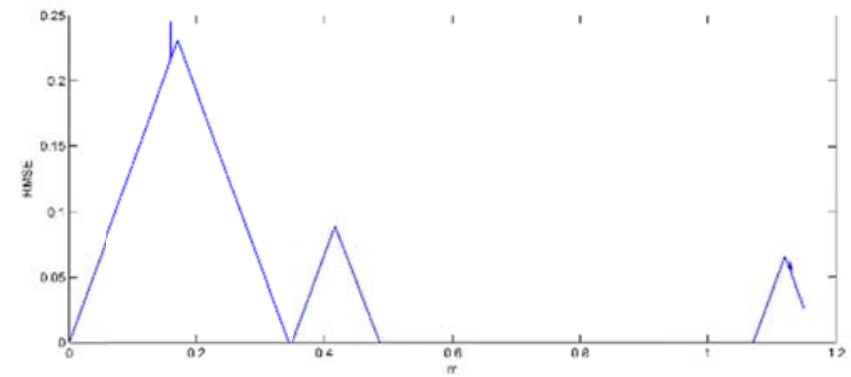

Figure $3 m-R M S E$

Observing that in Fig.3 RMSE is close to zero in the range [0.487,1.071] we can think it as the range of the switch angle which can be solved. Besides observing that RMSE is also close to zero when $\mathrm{m}$ is 0 and 0.379 , obviously we can let $\theta=0$ as the solution of the equation set when $\mathrm{m}$ is 0 . On the other hand we observe that RMSE also is possibly close to 0 when $\mathrm{m}$ is around 1.16 while it can not be satisfied on the right side of 1.07 and the left side of 1.16 . And this agrees with the conclusion of Fig.3.

The feasible range of $\mathrm{m}$ is too small around the isolated feasible points which are $0,0.379$ and 1.16. We let $R_{m}$ is $[0.487,1.071]$ for convenient calculation.

\section{The Relation of THD and $m$}

We enumerate $\mathrm{m}$ with the step length $1 \times 10^{-3}$ and solve corresponding $\theta_{1}, \theta_{2}$ and $\theta_{3}$ and the corresponding THD. The consequence is as follow:

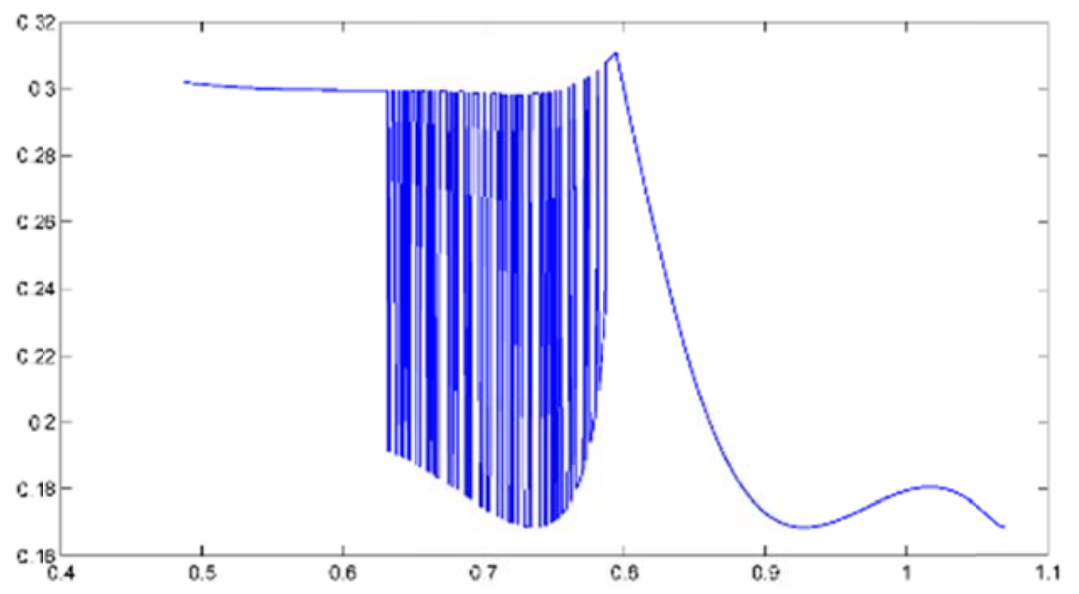

Figure $4 \mathrm{~m}$ - THD

Observing the Fig.4 knows that THD has large amplitude oscillation in [0.63 0.78] while in other part the curve is smoother. It grows $\mathrm{m}$ is bigger but THD is less. And the minimum $16.9 \%$ is in $\mathrm{m}=0.93$ nearby.

\section{Summary}

The Switching Angles are 0.5103, 0.9501 and 1.1255 by the nonlinear programming change for the equations and the initial setting. The rang of $\mathrm{m}$ is $[0.487,1.071]$ and the corresponding THD is $11.86 \%$ by consulting materials and enumerating $m$ and solving the equations. 


\section{References}

[1] Li L.Czarkowski D. , Liu Y. G. , and Pillay P. Multilevel selective harmonic elimination PWM technique in series-connected voltage inverters[C], in Proc. IEEE IAS, St, Louis, USA, 1998.

[2] Tolbert L M, Peng F Z, Cunnyngham T, et al. Charge balance control schemes for cascade multilevel converter in hybrid electric vehicles[J]. IEEE Trans. Ind. Electron. 2002, 49(5): 1058-1064.

[3] FEI Wan-min1, ZHANG Yan-li1, WANG Xue-hua2, et al. The inclusion relation of the solution of the SHEPWM problem for a multi level inverter. Proceedings of the CSEE. 2008. No.15, p.61-66.

[4] Guan Eryong, Song Pinggang, Ye Manyuan, et al. A method of solution to SHE-PWM switching angles for CSI[J]. Proceedings of the CSEE, 2005, 25(17), p. 62-65. 